\title{
Chemical evolution of C-Zn and r-process elements produced by the first generation stars
}

\author{
Yuhri Ishimaru* \\ Academic Support Center, Kogakuin University, 2665-1, Nakanomachi, Hachioji, Tokyo \\ 192-0015, Japan \\ E-mail: kt13121@ns.kogakuin.ac.jp

\section{Shinya Wanajo} \\ Department of Astronomy, Graduate School of Science, University of Tokyo, 7-3-1 Hongo, \\ Bunkyo-ku, Tokyo 113-8654, Japan \\ E-mail: wanajo@astron.s.u-tokyo.ac.jp
}

\section{Nikos Prantzos}

Institut d'Astrophysique de Paris, 98 bis, Boulevard Arago, 75014, Paris, France.

E-mail: prantzosdiap.fr

\begin{abstract}
Observed large scatters in abundance ratios of neutron-capture elements relative to iron in metalpoor stars may suggest incomplete mixing of the interstellar medium at the beginning of the Galaxy. On the other hand, recent studies of metal-poor stars show considerable small dispersions for abundance ratios of $\mathrm{C}-\mathrm{Zn}$. We discuss whether such variations of scatters in abundance ratios can be explained by a consistent chemical evolution model. We also attempt to constrain the origins of $r$-process elements, comparing predictions by an inhomogeneous chemical evolution model with new observational results with Subaru HDS.
\end{abstract}

International Symposium on Nuclear Astrophysics - Nuclei in the Cosmos - IX

June 25-30 2006

CERN, Geneva, Switzerland

${ }^{*}$ Speaker. 


\section{Introduction}

Metal-poor stars record enrichment history of the Galaxy at the early epoch. Abundance analysis of these stars reveals large star-to-star scatters in $r$-process elements [1, []. This may be interpreted as a result of incomplete mixing of the interstellar medium (ISM) at the beginning of the Galaxy. On the other hand, recent studies also show considerable small dispersions for abundance ratios of $\mathrm{C}-\mathrm{Zn}$ of extremely metal-poor stars [3]. It may seem to suggest that the ISM was already mixed completely when those stars were formed, contrary to interpretation of $r$-process elements. Therefore in this study, we discuss whether a consistent scenario can explain distributions of abundance ratios of lighter to heavier elements, using an inhomogeneous chemical evolution model based on several latest sets of supernova yields.

We also attempt to constrain the site(s) of $r$-process, comparing predictions from this model and new observational data of metal-poor stars. If metal-poor stars contain products of a single or a few supernovae ( $\mathrm{SNe}$ ), huge dispersions in abundances of $r$-process elements possibly imply that their yields are highly dependent on SN progenitor mass. However, no consensus about the origins of $r$-process elements has been achieved, although a few scenarios show some promise [4, 5]. In particular, observed enhancement of $\mathrm{Sr}$ comparing to $\mathrm{Ba}$ in metal-poor stars suggests the presence of the 'weak' $r$-process which produce mainly lighter $r$-elements. Thus we calculate enrichment of $\mathrm{Sr}, \mathrm{Pd}, \mathrm{Eu}$, and $\mathrm{Ba}$, and discuss bimodal origins of $r$-process.

\section{Bimodal $r$-process}

In our previous study, observed wide spread of Eu in metal-poor stars are shown to be wellreproduced by inhomogeneous enrichment scenario. In particular, sub-solar values of $[\mathrm{Eu} / \mathrm{Fe}]$ in stars of $[\mathrm{Fe} / \mathrm{H}] \sim-3$ can restrict the site of $r$-process as SNe of low-mass end stars such as $8-10 M_{\odot}$ [6]. Distribution of abundance ratio $[\mathrm{Ba} / \mathrm{Fe}]$ also supports this result.

However, recent comprehensive spectroscopic analyses of extremely metal-poor stars have further shown that lighter neutron-capture elements $(Z<56)$ significantly deviate from the scaled solar $r$-process pattern that fits to the heavier elements [7, 8, 9]. In particular, relative abundance of Sr exhibits wide variations not shared by $\mathrm{Ba}[12]$, suggesting that $\mathrm{Sr}$ at least does not come from a universal process. Although $\mathrm{Sr}$ is known to be produced also by weak $s$-process in massive stars, weak s-process yields at $[\mathrm{Fe} / \mathrm{H}] \sim-3$ cannot be enough high to exhibit the large enhancement of $\mathrm{Sr}$, since weak $s$-process requires a secondary element ${ }^{22} \mathrm{Ne}$ as neutron source. The second origin of $\mathrm{Sr}$ in metal-poor stars should be in primary source by massive stars. Thus we suggest bimodal $r$-processes for Sr. While only specific SNe produce heavier elements, e.g., Ba and $\mathrm{Eu}$, by main $r$-process, lighter elements such as $\mathrm{Sr}$ may be produced by residual $\mathrm{SNe}$ as 'weak' $r$-process.

Assuming the site of weak $r$-process as $\mathrm{SNe}$ of $20-30 M_{\odot}$ stars, we examine scatters in [ $\mathrm{Sr} / \mathrm{Ba}$ ] of metal-poor stars by an inhomogeneous chemical evolution model. As shown in fig. 1h, this scenario can well explain the observational distribution of [ $\mathrm{Sr} / \mathrm{Ba}$ ], when weak $r$-process produces $\sim 60 \%$ of Sr but only $\sim 1 \%$ of Ba in metal-poor stars [11].

\section{Origin of palladium}

Lighter $r$-process elements possibly have a distinct origin from heavier elements. However, the 

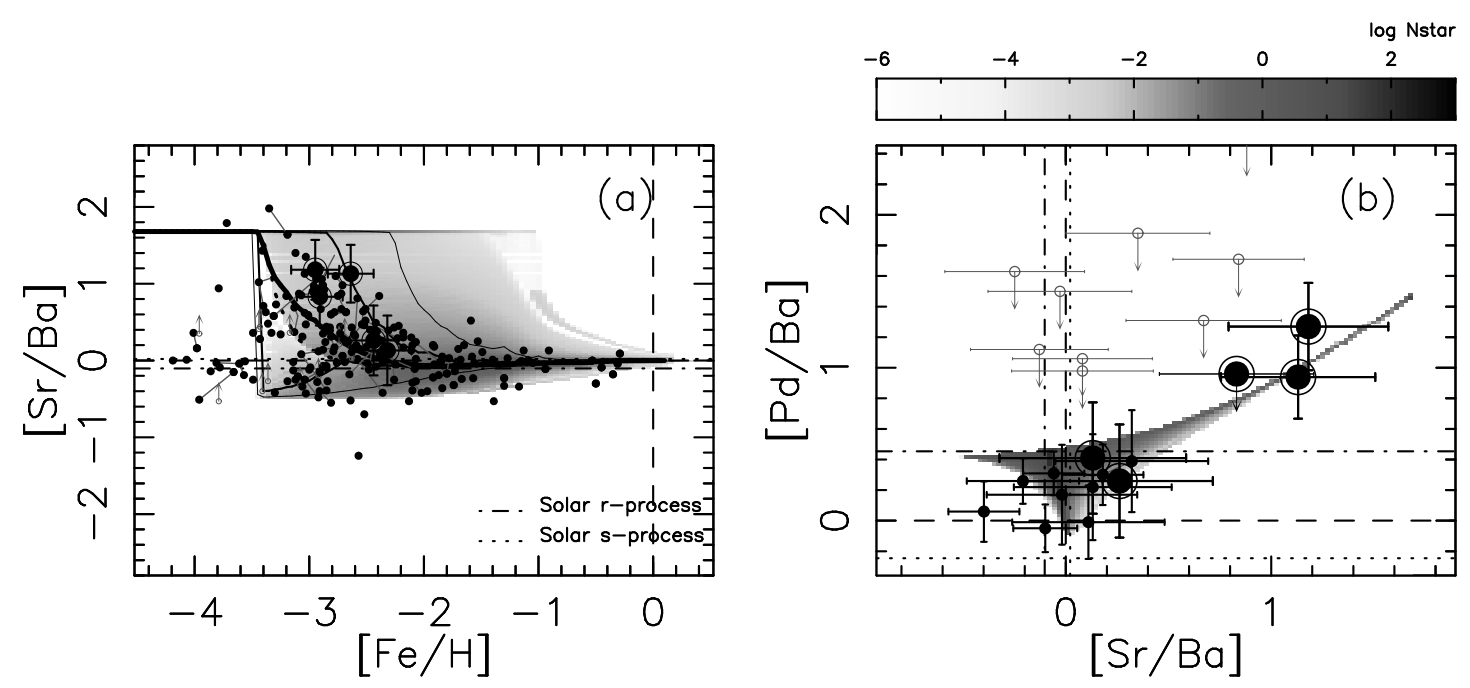

Figure 1: (a) $[\mathrm{Sr} / \mathrm{Ba}]$ vs. $[\mathrm{Fe} / \mathrm{H}]$ and $(\mathrm{b})[\mathrm{Pd} / \mathrm{Ba}]$ vs. [Sr/Ba] Gray-scale indicates predicted distribution of stellar fraction, using the Fe yield by Nomoto et al. (1997). The average stellar distributions are indicated by thick-solid lines with the 50\% (solid lines) and 90\% confidence intervals (thin-solid lines). Weak $r$-process fractions for $\mathrm{Sr}, \mathrm{Pd}$, and $\mathrm{Ba}$ are assumed as $60 \%, 10 \%$ and $1 \%$, respectively. The observational data of this study are given by large double circles, with other data (small circles).

location of the boundary in atomic number (or in mass number) between the two $r$-processes has been unresolved. Intermediate mass elements between $\mathrm{Sr}$ and $\mathrm{Ba}$ must provide clues to understand the nucleosynthesis of weak $r$-process. Thus, we suggest to use $\mathrm{Pd}$, which is located between typical light (e.g., Sr, Y, and Zr) and heavy (e.g., Ba, Eu, and Th) $r$-process elements, as diagnostics of the two $r$-processes.

We estimated Pd abundances for 5 metal-poor stars, using Subaru HDS. Figure $1 \mathrm{~b}$ show abundance ratios $[\mathrm{Pd} / \mathrm{Ba}]$ as a function of $[\mathrm{Sr} / \mathrm{Ba}]$. By definition, $[\mathrm{Sr} / \mathrm{Ba}]$ should increase with the fractional contribution of weak $r$-process to the stellar abundances. Thus, if Pd originates from weak $r$ like $\mathrm{Sr}$, i.e., its weak $r$ fraction is $\sim 60 \%$, [Pd/Ba] must show a correlation with the slope of unity to $[\mathrm{Sr} / \mathrm{Ba}]$. If $\mathrm{Pd}$ comes from main $r$-process like $\mathrm{Ba}$, $[\mathrm{Pd} / \mathrm{Ba}]$ must be constant irrespective of $[\mathrm{Sr} / \mathrm{Ba}]$. Our data show a mild correlation with the slope less than unity, suggesting that the weak $r$-process fraction for Pd takes intermediate value between those of $\mathrm{Sr}$ and $\mathrm{Ba}$. This trend is well explained by a model, assuming the weak $r$-process fraction for $\mathrm{Pd}$ as $\sim 10 \%$. Therefore, this result possibly implies that the weak $r$-process fraction is large at lighter elements, e.g., $\mathrm{Sr}$, but decreases with atomic mass towards heavier elements such as Ba and Eu. 
Recent observations result using Subaru shows that the abundance pattern of a metal-poor star HD 122563 is consistent with the above discussions: its abundance pattern gradually decreases with atomic number from $\mathrm{Sr}$ towards $\mathrm{Yb}[12]$. This star may be the first evidence for 'weak' $r$-process nucleosynthesis.

\section{Inhomogeneous chemical evolution of $\mathrm{O}-\mathrm{Zn}$}

In our inhomogeneous chemical evolution model, star formation is assumed to be induced through individual $\mathrm{SNe}$, and chemical compositions of new stars are given by the mass average of the remnant of the SN which induce their formation and the surrounding ISM. For comparison, we take three sets of SN yields; Woosley \& Weaver (1995)[14], Chieffi \& Limongi (2004)[13] and Nomoto et al. (2006)[15]. Using various SN yields, we calculate distributions of relative abundance ratios of $[\mathrm{O}-\mathrm{Zn} / \mathrm{Fe}]$ vs. $[\mathrm{Fe} / \mathrm{H}]$, and discuss implications of observational scatters in abundance ratios of metal-poor stars.

Figure 2 shows an example of $[\mathrm{A} 1 / \mathrm{Fe}]$ as a function of $[\mathrm{Fe} / \mathrm{H}]$. Although the nucleosynthesis of $\mathrm{Al}$ still contains significant uncertainties and the predicted distribution (gray-scale) is not completely consistent with observations (Fig. 2a ), the observed trend in metal-poor stars is suitable for discussion, where stars of $[\mathrm{Fe} / \mathrm{H}]<-3$ show a mild correlation with metallicity, with very small scatters. Typically, observations show largest scatters at $[\mathrm{Fe} / \mathrm{H}] \sim-3$. Model in fig. $2 \mathrm{~b}$ shows that stars formed from the zero-metal stars (circles) distribute at similar metallicity such as $[\mathrm{Fe} / \mathrm{H}] \sim-3$, but have large variations in abundance ratios relative to iron. This explains the observed largest scatters, suggesting that abundance ratios depends on stellar mass, while variation of iron productivity among different mass stars are not significant.

Chemical evolution model suggests that extremely metal-poor stars of $[\mathrm{Fe} / \mathrm{H}]<-3$ are also formed from gas containing products of zero-metal stars but much more efficiently diluted by the ISM. Since products of shorter life-time stars are diluted by less metal ISM, metallicity of new stars formed from such gas are more reduced, but relative abundance ratios are not affected by efficiency of dilution. As a result, the mass dependency of yields of metal-poor stars is elongated towards metal-poorer region by dilutions, and therefore, a trend of $[\mathrm{X} / \mathrm{Fe}]$ vs. $[\mathrm{Fe} / \mathrm{H}]$ with small scatter appear naturally.

Hypernovae can be the other possibility to account for trends with small scatters in metal-poor stars. Figure 3 show the cases of $[\mathrm{Mn} / \mathrm{Fe}]$ and $[\mathrm{Zn} / \mathrm{Fe}]$ predicted by a model with Nomoto et al. (2006) [15], in which all stars heavier than $20 M_{\odot}$ are assumed to be hypernovae. Since the explosion energy of hypernovae is larger than that of SNe, SNR expands wider and is diluted efficiently by the ISM much more than the case of SNe. Therefore, stars formed from HNe distribute at $[\mathrm{Fe} / \mathrm{H}] \sim-4$ and the difference of yields between $\mathrm{SNe}$ and $\mathrm{HNe}$ causes abundance trends seen in metal-poor stars with $[\mathrm{Fe} / \mathrm{H}]$ lower than -3 .

In conclusion, it is basically possible to reproduce the observed variations in scatters and trends in various elements, although up to now, no SN models can completely explain the trends and scatters in chemical abundances of all elements. Therefore, observational data must give strong constraints on future works of SN models. 

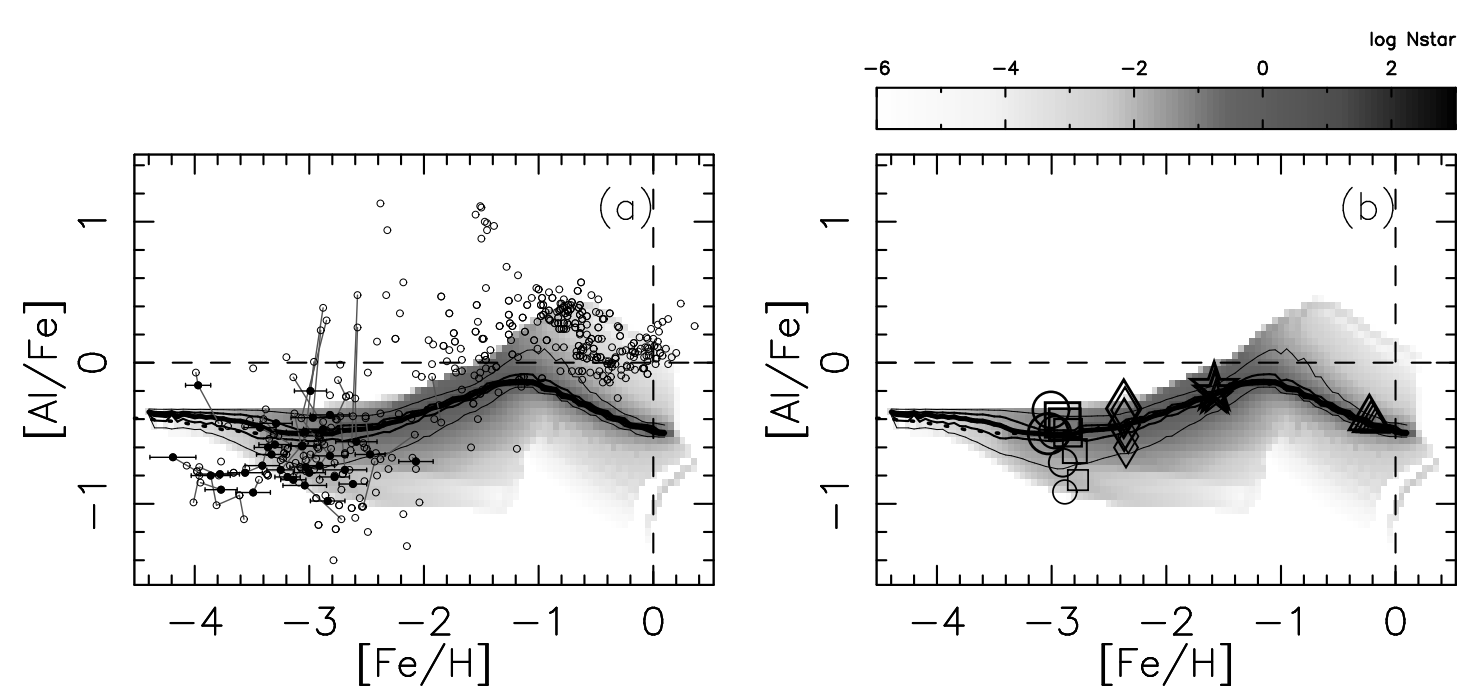

Figure 2: $[\mathrm{A} 1 / \mathrm{Fe}]$ as a function of $[\mathrm{Fe} / \mathrm{H}]$. Gray-scale indicates distribution of stellar fraction predicted by a model using the data of supernova yield by Chieffi \& Limongi (2004). Solid lines are the same with Fig. 1. (a) Comparison of the prediction with observational data of Cayrel et al. (2004) denoted by small filled circles and other data (small open circles). Stars formed through supernova from progenitors of zero metal (circles), $5 \times 10^{-5} Z_{\odot}$ (squares), $5 \times 10^{-3} Z_{\odot}$ (diamonds), $5 \times 10^{-2} Z_{\odot}$ (stars), and $0.3 Z_{\odot}($ triangles) are indicated in (b) The size of symbols increase with progenitor masses from $13 M_{\odot}$ to $35 M_{\odot}$.
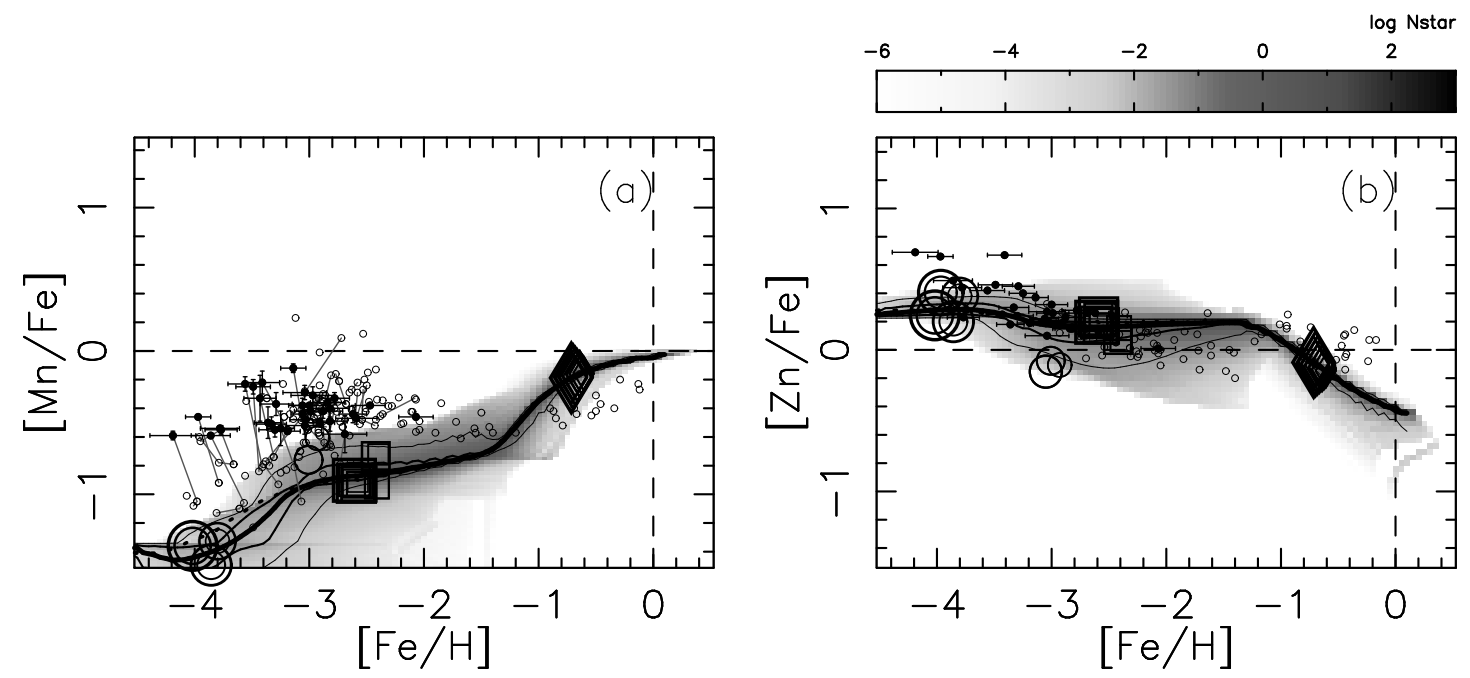

Figure 3: a) $[\mathrm{Mn} / \mathrm{Fe}]$, and b) $[\mathrm{Zn} / \mathrm{Fe}]$ as functions of $[\mathrm{Fe} / \mathrm{H}]$. Gray-scale indicates distribution of stellar fraction predicted by the model with the data of supernova yield by Nomoto et al. (2006), in which all stars heavier than $20 M_{\odot}$ are assumed to be hypernovae. Solid lines are the same with Fig. 1. Observational data are taken from Cayrel et al. (2004, small filled circles) and others (small open circles). Stars formed through supernova from progenitors of zero metal, $5 \times 10^{-3} Z_{\odot}$, and $0.2 Z_{\odot}$ are indicated by circles, squares, and diamonds, respectively. Double symbols denote stars formed from hypernovae. The size of symbols increase with progenitor masses from $13 M_{\odot}$ to $40 M_{\odot}$. 


\section{Conclusions}

Origins of $r$-process elements are constrained by comparing predictions from an inhomogeneous chemical evolution model with chemical compositions of extremely metal-poor stars obtained by Subaru HDS. Sub-solar values of $[\mathrm{Eu} / \mathrm{Fe}]$ in three metal-poor stars imply the site of main $r$-process to be low-mass end of the SN mass range. Enhancements of Sr comparing to heavier elements suggest existence of two distinct sources for $r$-process. New data of Pd abundance suggest that 'weak' $r$-process yield is large at around $\mathrm{Sr}$, but decreases with atomic mass towards heavier elements such as Ba and Eu.

It is also shown that variations of scatters in observed trends of abundance ratios of $\mathrm{C}-\mathrm{Zn}$ with metallicity can be understood by inhomogeneous chemical evolution scenario. The maximum dispersion at $[\mathrm{Fe} / \mathrm{H}] \sim-3$ comes from stellar mass-dependencies of yields of zero-metal or extremely metal-poor stars. Stars formed from the highly diluted gas containing products of $\mathrm{SNe}$ or $\mathrm{HNe}$ of zero-metal stars cause the trend with small scatters shown in stars of $[\mathrm{Fe} / \mathrm{H}]<-3$.

\section{References}

[1] Ryan, S. G., Norris, J. E., \& Beers, T. C. 1996, ApJ, 471, 254

[2] Honda, S., Aoki, W., Kajino, T., et al., 2004, ApJ, 607, 474

[3] Cayrel, R., et al., 2004, A\&A, 416, 1117

[4] Woosley, S. E., Wilson, J. R., Mathews, G. J., et al. 1994, ApJ, 433, 229

[5] Wanajo, S., Tamamura, M., Itoh, N., et al. 2003, ApJ, 593, 968

[6] Ishimaru, Y., Wanajo, S., Aoki, W., \& Ryan, S. G., 2004, ApJ, 600, L47

[7] Hill, V., Plez, B., Cayrel, R., et al. 2002, A\&A, 387, 560

[8] Cowan, J. J., Sneden, C., Burles, S.,et al. 2002, ApJ, 572, 861

[9] Sneden, C., Cowan, J. J., Lawler, J. E., et al. 2003, ApJ, 591, 936

[10] Nomoto, K., Hashimoto, M., Tsujimoto, T., et al. 1997, Nucl. Phys. A, 616, 79

[11] Ishimaru, Y. \& Wanajo, S. 2000, in First Stars, ed. A. Weiss, T. Abel,\& V. Hill (Springer), p.189

[12] Honda, S., Aoki, W., Ishimaru, Y., Wanajo, S., Ryan, S. G., 2006, ApJ in press.

[13] Chieffi, A., Limongi, M., 2004, ApJ, 608, 405

[14] Woosley, S. E., Weaver, T. A., 1995, ApJS, 101, 181

[15] Nomoto, K., Umeda, H., Tominaga, N., et a., 2006, in preaprattion 